\title{
Nurse Administered Propofol Sedation (NAPS) versus On-call Anesthesiologist Administered Propofol Sedation (OAPS) in Elective Colonoscopy
}

\author{
Kasenee Tiankanon $^{1,2}$, Parit Mekaroonkamol ${ }^{1,2}$, Rapat Pittayanon ${ }^{1,2}$, Pradermchai Kongkam ${ }^{2,3}$, Sutep Gonlachanvit ${ }^{2,4}$, \\ Rungsun Rerknimitr ${ }^{1,2}$
}

1) Center of Excellence for Innovation and Endoscopy in Gastrointestinal Oncology, Chulalongkorn University, Bangkok;

2) Division of Gastroenterology, Faculty of Medicine, Chulalongkorn University and King Chulalongkorn Memorial Hospital, Thai Red Cross, Bangkok;

3) Pancreas Research Unit, Department of Medicine, Faculty of Medicine, Chulalongkorn University, Bangkok;

4) Center of Excellence on Neurogastroenterology and Motility, Department of Medicine, Faculty of Medicine, Chulalongkorn University, Bangkok, Thailand

Address for correspondence: Rungsun Rerknimitr, MD, FRCP (London), FASGE Professor of Medicine Division of Gastroenterology, Department of Medicine, Faculty of Medicine, Chulalongkorn University and King Chulalongkorn Memorial Hospital, Rama 4 Road, Patumwan, Bangkok, Thailand 10330 ercp@live.com

\section{ABSTRACT}

Background \& Aims: As on-call anesthesiologist administered propofol sedation (OAPS) is costly and not readily available in all endoscopy units, endoscopy nurse administered propofol sedation (NAPS) can be an effective alternative. This study aimed to compare the dosage of propofol used by NAPS versus OAPS, cardiopulmonary adverse events and recovery time in low risk patients undergoing outpatient elective colonoscopy.

Methods: A retrospective propensity score-matched cohort study was conducted. Electronic medical records of elective colonoscopies performed by 3 experienced endoscopists from January 2016 to December 2019 were retrieved. OAPSs were performed by 10 certified anesthesiologists while NAPSs were performed by 8 experienced registered endoscopy nurses. Baseline characteristics, performing endoscopist, cecal intubation time, withdrawal time, propofol dosage per procedure, and adverse events were collected and analyzed using 3:1 (NAPS:OAPS) propensity score matching by age, performing endoscopist and difficulty of colonoscopy as co-variates with standardized mean deviation of $<0.1$.

Results: 278 eligible patients were included. After propensity score matching, there were 189 patients in NAPS and 63 in OAPS group for analysis. Demographic data were not different between the two groups. All procedures were technically successful with no difference in cecal intubation time $(6.0 \pm 4$ min vs $6.8 \pm 4$ $\min ; \mathrm{p}=0.13)$ or total procedural time $(17.2 \pm 16 \mathrm{~min}$ vs $16.3 \pm 6 \mathrm{~min} ; \mathrm{p}=0.66)$. Propofol dosage $/ \mathrm{kg} /$ hour were significantly lower in the NAPS group, $(11.4 \pm 4 \mathrm{mg} / \mathrm{kg} /$ hour vs. $16.6 \pm 8 \mathrm{mg} / \mathrm{kg} / \mathrm{hour} ; \mathrm{p}<0.001)$. There were less minor cardiopulmonary adverse events in NAPS when compared to the OAPS group ( $2.2 \%$ vs $4.7 \%$; $\mathrm{p}=0.014)$. Conclusions: NAPS in elective colonoscopy in low-risk patients is as effective as OAPS but requires a significant lower dosage of propofol. Minor cardiopulmonary adverse events were recorded in the NAPS group compared to OAPS.

Key words: propofol - sedation - colonoscopy - NAPS - nurse administered sedation.

Abbreviations: ASA: American Society of Anesthesiologists; CAE: cardiopulmonary adverse event; NAPS: nurse administered propofol sedation; OAPS: on-call anesthesiologist administered propofol sedation.

\section{INTRODUCTION}

Intravenous propofol has become the preferred sedating agent for low risk patients undergoing non-advanced gastrointestinal endoscopic procedures such as elective colonoscopy due to its rapid onset, easy titratability, and short duration of action [1, 2]. Various studies including meta-analyses, comparing the safety and efficacy of propofol sedation with conventional methods (narcotics and/or benzodiazepines), have shown the superiority of propofol in terms of shorter recovery time and patient's and endoscopist's satisfaction [3-6].

Endoscopy nurse administered propofol sedation (NAPS), in which propofol is administered as a single agent to target moderate to deep sedation under the direction of the endoscopists, has demonstrated high level of safety and efficacy $[7,8]$. Nurse administered propofol sedation also has been associated with shorter sedating durations and recovery time, with better patient's satisfaction while maintaining the same level of sedation, compared to the conventional medication (combination of an opioid and/or benzodiazepine) [8].

However, the subject of NAPS has raised great controversy and debate. Due to a narrow therapeutic window of propofol, many institutions have restricted its use only to be provided 
by anesthesiologists or certified nurse anesthetists [9]. The concern is that inadequate experience in recognizing and managing cardiopulmonary adverse events associated with propofol used, especially hypoventilation, obstructed airway and cardio-circulatory depression can potentially compromise the patient's safety $[10,11]$. As a result, the practice of NAPS is not widely accepted in some countries such as the United States and certain countries in Asia [9, 12-14]. In these countries, propofol-based sedation for patients undergoing routine endoscopic procedures is often limited by the availability of anesthesia personnel [15-17].

Recent large population-based studies reported no significant difference in unplanned cardiopulmonary events in patients receiving sedation administered by endoscopists when compared to anesthesiologists during colonoscopy $[18,19]$. However, the adverse events risk was higher in the anesthesiologist-administered group during upper endoscopies [18]. In addition, a recent study reported a higher risk of colonic perforation rate under anesthesia services [20]. Despite a huge cost advantage, avoiding anesthesia fees, an increasing evidence to support the safety, feasibility and patients' satisfaction of NAPS [21, 22], propofol use is still restrictive and was used in only $2.9 \%$ of colonoscopies when an anesthesiologist was not available [18].

Hypothetically, the dosage of propofol given by the endoscopy nurse might be lower than that of given by the oncall anesthesiologist administered propofol sedation (OAPS) since the endoscopy nurse is more familiar with certain steps during colonoscopy when propofol requirement may different. For instance, the dosage might be increased during sigmoid intubation due to pressure discomfort developed from colonoscope pushing whereas during colonoscope withdrawal the requirement of dosage could be reduced.

To date, data comparing the safety and efficacy of propofol used between NAPS and OAPS is very limited. This study aimed to compare the dosage of propofol used by NAPS versus OAPS, cardiopulmonary adverse events (CAEs) and recovery time in low risk patients undergoing outpatient elective colonoscopy.

\section{METHODS}

A retrospective propensity score-matched cohort study was conducted. Electronic medical records were retrieved to identify a cohort of patients aged 18-90 years with the American Society of Anesthesiologists (ASA) class I or II who had undergone an elective outpatient colonoscopy by 3 experienced endoscopists (R.R., P.K., S.G.) using propofolbased sedation between January $1^{\text {st, }} 2016$ and December $31^{\text {st }}$, 2019 at the King Chulalongkorn Memorial hospital. Subjects who were pregnant, ASA class III or IV or had allergy to propofol or its component (soybeans and eggs) were excluded.

All eligible patients were included. Baseline demographic data, duration of procedure, performing endoscopists, amount of sedation used, detailed endoscopic findings, and adverse events during and after colonoscopy were collected and compared between the NAPS and OAPS group.

Due to the lack of routinely available dedicated anesthesiologists in our endoscopy unit, the number of cases with on-call anesthesiologist administered propofol sedation
(OAPS) was therefore limited, hence the higher number of NAPS in the routine practice of our institution.

With unequal distribution of both groups due to the limited number of OAPS, a propensity score matching ratio of 3:1 (NAPS: OAPS) was performed in order to reduce the sedation assignment bias and mimic randomization. Patients' age, performing endoscopist, and difficulty of colonoscopy (defined by procedures with more than 3 polypectomies, polyp size more than $3 \mathrm{~cm}$ and cecal intubation time more than 20 minutes) were accounted as co-variates with the acceptable standardized mean deviation of less than 0.1 .

This study protocol was conducted in accordance with the Helsinki Declaration and was approved by our institutional Ethical Committee with an IRB number 136/60.

Verbal and written informed consent for sedation were obtained from all patients before the procedures. All colonoscopies were performed in the left lateral decubitus position. An infusion catheter for propofol administration was placed on the subject's forearm shortly before the examination started and was removed once the standard discharge criteria were met after completion of the procedure. Every subject received nasal oxygen canula 3-5 litre per min before and during sedation. During the procedure, all patients were observed with standard monitoring which included automated non-invasive measuring of blood pressure every 3-5 minutes and continuous assessment of peripheral oxygen saturation and heart rate. In addition to monitoring of vital signs, the subjects' condition such as adequate rise of chest wall, signs of apnea, skin and lip coloration, amount of secretion, etc. was assessed globally by visual inspection.

On-call anesthesiologist administered propofol sedation was performed by 10 board-certified anesthesiologists who were experienced in gastrointestinal endoscopy sedation. They performed sedation only when the patients specifically requested for this service. The attending anesthesiologist performed all sedation him/herself without the nurse anesthetists. Dosage and method of propofol administration, such as intermittent bolus or continuous infusion with or without other concomitant sedative depended upon the discretion of expert anesthesiologist in the OAPS group.

Endoscopy nurse administered propofol sedation was performed by 8 registered endoscopy nurses. All patients in the NAPS group received propofol as a single sedative agent with a standardized protocol using a bolus dose of $0.5-1 \mathrm{mg} /$ $\mathrm{kg}$, then titrated to $0.25-0.5 \mathrm{mg} / \mathrm{kg} /$ hour with infusion pump until moderate sedation level was achieved, defined as: (1) the subject responds purposefully to verbal commands; (2) airway of the subject is patent, and spontaneous ventilation is adequate; and (3) cardiovascular function is unaffected [23]. When target level was not reached, an additional bolus of 0.5 $\mathrm{mg} / \mathrm{kg}$ up to a maximum of $1 \mathrm{mg} / \mathrm{kg}$ per dose in $1 \mathrm{~min}$ interval of propofol could be administered.

All nurses in NAPS group underwent a training for this sedation protocol prior to the study. The nurses' program comprised of a 6-week course of both theoretical and handson training in airway management under supervision of an experienced nurse and certified endoscopists [24]. The nurse was dedicated solely to propofol administration and patient monitoring, while another nurse or technical assistant provided 
procedural assistance to the endoscopist. Similar to OAPS, NAPS was an optional for the patients to select it if their condition allowed.

Procedural duration was defined as the time from the initial insertion of the colonoscope to its complete withdrawal. Time needed to reach the cecum was also recorded. Adverse events such as respiratory suppression, agitation, aspiration were captured as per standard protocol by visual inspection of the monitor and recorded on the vital sign sheet.

After colonoscopy, each subject was transferred to the recovery room and monitored by another dedicated nurse. Full recovery, time to regain consciousness, and psychomotor activity, were evaluated using 2 criteria: level of consciousness (full awareness and appropriately responding to questions) and the ability to walk in a straight line without instability for 5 meters. After full recovery was confirmed, the patient was discharged with assistance of his/her designated caretaker. If a full recovery was not obtained within 1 hour, the endoscopist would be notified to evaluate the patient at bedside and the subjects were required to extend their standard monitoring in the endoscopic until full recovery was confirmed.

The primary outcome was the difference in propofol dosages $/ \mathrm{kg} /$ hour used by NAPS compared to OAPS. The secondary outcomes were: 1) success rate of the procedures, defined by successful cecal intubation and the absence of premature abortion of the procedure, 2) the occurrence of CAE (minor to intermediate and sentinel), as defined by the World Society of Intravenous Anaesthesia (SIVA) International Sedation Task Force [25] during and after the procedure, and during recovery time.

Minor to intermediate events were hypoxia, defined as oxygen saturation of $75-90 \%$ that lasted for $20-60$ seconds, transient ( $<60$ seconds) apnea, transient airway obstruction requiring air way repositioning, allergic reaction, failed sedation, bradycardia or tachycardia ( $>25 \%$ change from baseline heart rate), and seizure. Blood pressure below 90/60 $\mathrm{mmHg}$ or a drop-in blood pressure from baseline of more than $50 \mathrm{mmHg}$ systolic or $25 \%$ in mean arterial pressure after sedation was defined as hypotension associated with sedation which was included in minor to intermediate adverse events. Sentinel or major events included prolonged ( $>60$ seconds) hypoxia or oxygen desaturation of less than $75 \%$, prolonged apnea (>60 seconds), cardiovascular shock, and cardiac arrest.

All adverse events were managed with immediate cessation of propofol infusion, increased oxygen flow (5-7 L/min), and saline intravenous bolus if needed until the patient became less sedated. Airway obstruction was treated with chin lift and jaw thrust. If the subject started to gain consciousness during the procedure and vital signs were stable, propofol would then be re-administered. Nasopharyngeal airway placement, mask ventilation, or endotracheal intubation was not required in any colonoscopy. No laryngospasm or threatening laryngospasm were observed during the procedures.

The analyses were done using IBM SPSS Statistics for Windows, Version 22.0 (IBM Corp., Armonk, NY). Propofol dosages were compared between groups using the student t-test. Descriptive data were presented as frequency and percentage. Baseline characteristics were calculated and presented as mean \pm standard deviation for parametric data. A value of $\mathrm{p}<0.05$ was considered statistically significant. Other qualitative variables were recorded as number (percent) and mean. Comparing qualitative data between two groups using chi-square or Fisher's exact test. Median, inter-quantile range and range were used to report the quantitative variables and comparing between the two groups using the Mann-Whitney U test.

\section{RESULTS}

A total of 766 patients underwent elective colonoscopy by 3 experienced endoscopists during the study period. Among these, 488 did not use propofol-based sedation, leaving 278 patients to remain in the study. 214 patients were in the NAPS group, while 64 patients were in the OAPS group. After applying 3:1 propensity score matching using the patient's age and performing endoscopist as co-variates with standardized mean deviation of less than 0.1 , a total of 189 patients in the NAPS group and 63 patients in the OAPS group were included for analysis (Fig. 1). Baseline characteristic, cecal intubation time, withdrawal time, propofol dosage administered, and adverse events were compared between the two groups (Table I).

The patients' mean age was $65.2 \pm 12$ years (range $20-85$ years). The most common indications for elective colonoscopy were for colorectal cancer screening (85\%). Demographic data including clinically relevant endoscopic findings that might increase difficulties of the endoscopic procedure were not different between both groups as shown in Table I.

All procedures in both groups were completed with 100\% successful cecal intubation rate. There was no unplanned abortion of the procedure. Overall, mean procedural time was approximately 17 minutes with no difference in cecal intubation time or withdrawal time between both groups (Table II).

In the OAPS group, 8 out of 63 subjects received additional sedative concomitantly with propofol-based sedation. Four patients received $2.5 \mathrm{mg}$ of midazolam and $25 \mathrm{mg}$ of meperidine, 3 patients received $25 \mathrm{mg}$ of fentanyl, and 1 received $50 \mathrm{mg}$ fentanyl. The remaining 55 patients in the OAPS group received propofol as single sedative agent.

The propofol dosage $/ \mathrm{kg} / \mathrm{hr}$ was significantly lower in the NAPS group than the OAPS group $(11.4 \pm 4 \mathrm{mg} / \mathrm{kg} / \mathrm{hour}$ vs. $16.6 \pm 8 \mathrm{mg} / \mathrm{kg} /$ hour; $\mathrm{p}<0.001$ ) (Table II).

To account for confounding factors that may increase the propofol dosage required during colonoscopy, a subgroup analysis of typical colonoscopies excluding those with difficult cecal intubation, procedures with more than 3 polypectomies, and those with large polyp of more than $3 \mathrm{~cm}$ was performed. The results remained consistent with a lower propofol dosage used in the NAPS group $(11.9 \pm 4 \mathrm{mg} / \mathrm{kg} /$ hour vs. $17.0 \pm 8 \mathrm{mg} /$ $\mathrm{kg}$ /hour, $\mathrm{p}<0.001$ ) (Table III).

No serious adverse events occurred. Minor CAE rate was lower in NAPS, compared to the OAPS group $(2.1 \%$ vs $4.8 \% ; \mathrm{p}=0.014$ ). Four patients in the NAPS group developed mild hypoxia that required chin lift maneuver $(n=2)$ and transient hypotension requiring crystalloid bolus intravenously $(n=2)$, while three CAEs in the OPAS group were transient hypotension requiring crystalloid intravenous bolus $(n=1)$ and hypotension requiring bolus ephedrine injection $(n=3)$. One 


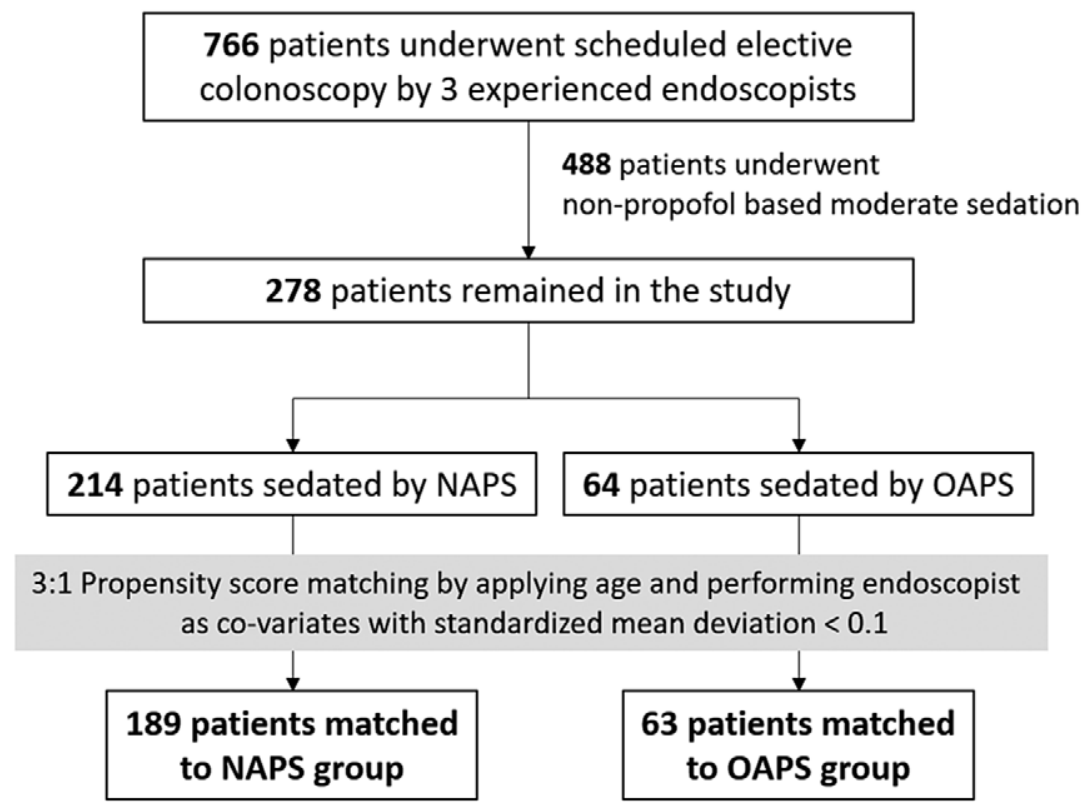

Fig. 1. Patients flow chart. NAPS: nurse administered propofol sedation; OAPS: on-call anesthesiologist administered propofol sedation.

CAE occurred in a patient sedated with fentanyl and propofol combination

Recorded time to recovery after the procedure was missing in $23 / 189$ cases $(12.1 \%)$ in the NAPS group and $13 / 63$ cases (20.6\%) in the OAPS group. However, the remaining patients with available data (166 in NAPS group and 50 in OAPS group) all had a full recovery within 60 minutes after the procedure. $\left(\chi^{2}=2.78, p=0.43\right)$

\section{DISCUSSION}

Propofol has gained popularity in low-risk endoscopic procedures over the past decade $[26,27]$. However, the theoretical risk of propofol being administered by non- anesthesiological personnel has greatly limited its use even though emerging studies have demonstrated favorable safety and patient's satisfaction of NAPS [26-30]. One of the reasons for such reluctance to adopt this practice is perhaps the lack of studies directly comparing NAPS and OAPS.

This study emphasized the safety of propofol under NAPS in routine endoscopy practice outside of a trial setting. Our study demonstrated that colonoscopy can be performed safely and effectively in selected low-risk patients (ASA 1 and 2) using propofol sedation administered by trained endoscopy nurses according to a protocol established in collaboration with endoscopists and anesthesiologists. While achieving similar colonoscopy success rate, there were no significant differences in the quality indicators of the colonoscopy, including adverse

Table I. Baseline characteristic of patients

\begin{tabular}{llcccc}
\hline Characteristics & & $\begin{array}{c}\text { Total } \\
\mathrm{n}=252\end{array}$ & $\begin{array}{c}\text { NAPS } \\
\mathrm{n}=189\end{array}$ & $\begin{array}{c}\text { OAPS } \\
\mathrm{n}=63\end{array}$ & $\mathrm{p}$ \\
\hline Gender & Male N, (\%) & $145(57.5 \%)$ & $106(56 \%)$ & $39(62 \%)$ & 0.42 \\
& Female N, (\%) & $107(42.5 \%)$ & $83(44 \%)$ & $24(38 \%)$ & \\
Age (years) & Mean \pm SD & $65.2 \pm 12$ & $65.2 \pm 12$ & $65.2 \pm 12$ & 0.99 \\
Body weight (kg) & Mean \pm SD & $64.6 \pm 12$ & $64.9 \pm 11$ & $63.3 \pm 12$ & 0.31 \\
Indications & CRC screening & $213(85 \%)$ & $159(84 \%)$ & $54(85 \%)$ & 0.06 \\
& Clinically stable recent & $9(4 \%)$ & $5(3 \%)$ & $4(6 \%)$ & \\
& GI bleeding & $19(7 \%)$ & $16(8 \%)$ & $3(5 \%)$ & \\
& Constipation & $11(4 \%)$ & $9(5 \%)$ & $2(4 \%)$ & \\
& Others & $35(13 \%)$ & $28(14 \%)$ & $7(11 \%)$ & 0.46 \\
& Diverticulosis & $5(2 \%)$ & $4(2 \%)$ & $1(1.6 \%)$ & 0.19 \\
& $\begin{array}{l}\text { Polyp larger than 3 } \\
\text { Endoscopic findings }\end{array}$ & & & \\
& More than 3 polyps & $21(8.3 \%)$ & $16(8.5 \%)$ & $5(7.9 \%)$ & 0.9 \\
& per procedure & & & & \\
\hline
\end{tabular}

CRC: colorectal cancer; GI: gastrointestinal; NAPS: nurse administered propofol sedation; OAPS: on-call anesthesiologist administered propofol sedation. 
Table II. Comparing procedural duration, propofol dosage used and adverse events between groups

\begin{tabular}{lccc}
\hline Parameters & $\begin{array}{c}\text { NAPS } \\
\mathrm{n}=189\end{array}$ & $\begin{array}{c}\text { OAPS } \\
\mathrm{n}=63\end{array}$ & $\mathrm{p}$ \\
\hline Propofol dosage/bodyweight/time (mg/kg/hr.) & $11.4 \pm 4$ & $16.6 \pm 8$ & $<0.001$ \\
Dosage of propofol/body weight (mg/kg) & $2.8 \pm 2$ & $4.0 \pm 2$ & $<0.001$ \\
Total dosage of propofol (mg/procedure) & $180.2 \pm 102$ & $248.4 \pm 88$ & $<0.001$ \\
Procedural success rate & 100 & 100 & 1.0 \\
Cecal intubation time (min) & $6.0 \pm 4$ & $6.8 \pm 4$ & 0.13 \\
Withdrawal time (min) & $11.2 \pm 15$ & $9.4 \pm 6$ & 0.37 \\
Total procedure time (min) & $17.2 \pm 16$ & $16.3 \pm 6$ & 0.66 \\
Cardiopulmonary adverse events, cases N, (\%) & $4(2.1)$ & $3(4.8)$ & 0.014 \\
\hline
\end{tabular}

For abbreviations see table I.

Table III. Comparing propofol dosage used and adverse events between groups after excluding complex procedures.

\begin{tabular}{lccc}
\hline Parameters & $\begin{array}{c}\text { NAPS } \\
(\mathrm{n}=172)\end{array}$ & $\begin{array}{c}\text { OAPS } \\
(\mathrm{n}=57)\end{array}$ & $\mathrm{p}$ \\
\hline Propofol dosase/bodyweight/time $(\mathrm{mg} / \mathrm{kg} / \mathrm{hr})$. & $11.9 \pm 4$ & $17.0 \pm 8$ & $<0.001$ \\
Dosage of propofol/body weight $(\mathrm{mg} / \mathrm{kg})$ & $2.7 \pm 1$ & $4.1 \pm 2$ & $<0.001$ \\
Total dosage of propofol (mg/procedure) & $168.7 \pm 59$ & $246.3 \pm 80$ & $<0.001$ \\
Cardiopulmonary adverse events, cases (\%) & $3(1.7 \%)$ & $3(5.2 \%)$ & 0.005 \\
\hline
\end{tabular}

events rate and procedural time between groups. The only intervention that was different between both groups was the administration of bolus ephedrine in the OPAS group, as only anesthesiologists are allowed to inject ephedrine in our institution.

Interestingly, more conservative dosage of propofol was observed in NAPS groups $(11.4 \pm 4 \mathrm{mg} / \mathrm{kg} /$ hour vs. $16.6 \pm 8 \mathrm{mg}$./ $\mathrm{kg} /$ hour; $\mathrm{p}<0.001$ ). This difference may be explained by the familiarity of endoscopic procedure among endoscopy nurses providing NAPS. Exclusion of complex procedures, defined as those with difficult cecal intubation, procedures with more than 3 polypectomies, and those with 1 larger polyp than $3 \mathrm{~cm}$ allowed us to limit the confounding factors of prolonged air insufflation, special equipment used, or a different polypectomy technique, leaving the results to reflect more of routine low-risk colonoscopies. Consistent results after subgroup analysis also highlighted the feasibility and practicality of NAPS.

With a rotating schedule of anesthesiologists in multispecialty procedures, the familiarity of the technical steps of a colonoscopy can be limited when compared to dedicated experienced endoscopy nurses or endoscopists. As opposed to typical surgery, endoscopic procedure is relatively quick with a short turnaround time with a predictable procedural course i.e. where the scope may cause pain and when the procedure is comfortable for the patient. Required level of sedation is relatively operator-dependent in certain parts of the procedure; for example, an experienced endoscopist can identify the area of looping of the scope and would be able to anticipate an increase in requirement of sedation or to decrease it when the scope is being withdrawn. Although endoscopists are encouraged to notify the entire endoscopy staff regarding the estimated remaining procedural time, endoscopy nurses can typically make this observation independently. Therefore, endoscopist-directed sedation may have the advantage over anesthesiologist-administered approach in routine low-risk endoscopy. This lower required dosage of propofol may also explain the lower CAEs observed in the NAPS group in our study.

As the procedural time and recovery time were similar in both NAPS and OAPS, the direct cost difference is determined by the difference in propofol dosages used and the fee for an additional anesthesiology service. Considering the propofol cost alone, in OAPS group, the cost was 1.46 times higher compared to the NAPS group, and the cost for the anesthesiologist's presence could be much higher. In 2005, it was estimated the cost incurred from the anesthesiologist fee alone for all colonoscopy in the United States, had they all been done with the presence of an anesthesiologist, would have been more than 1.5 billion US dollars per year [31]. Moreover, by deploying non-anesthesia personnel for propofol sedation, the already scarce anesthesia resources could be allocated for patients with more complex conditions. Although the economic benefit of not requiring an anesthesiologist for endoscopic procedures is obvious, further substantiating research on cost analysis is warranted.

The propensity-score matched analysis also helped balance the important baseline characteristics of the patients in two groups. In addition, unlike the prospective design, our retrospective cohorts were more representative of a "realworld" practice and were not subject to the Hawthorne effect, where the sedation pattern may be affected by the notion of being monitored. 
The limitation of the study was the nature of the retrospective study design, which is subject to missing data and uncontrolled confounding factors. However, a meticulous propensity score matching was performed to overcome these limitations and reduce the assignment bias, mimicking randomization. We used the patients' age, performing endoscopist, and difficulty of colonoscopy as co-variates with the acceptable standardized mean deviation of less than 0.1 , which we believe has lessened the confounding factors significantly.

Due to the limited availability of anesthesiology service at our institution, the sample size of both groups were inherently different and was relatively small in OAPS group. This might impact the power to detect any major cardiorespiratory adverse events, which was estimated only at $0.01 \%$ for propofol sedation [21].

The sedation protocol in OAPS group that allowed the anesthesiologist to administer other sedatives/analgesics at his or her discretion posed the risk of inter-operator variations and could have limited the generalization of our results.

Another limitation was the lack of continuous capnography monitoring during the procedures as it was not included in our institutional standard monitoring protocol during the study period. Nevertheless, we believe that it is unlikely that the results would be different due to our intensive procedural monitoring protocol and only a moderate depth of sedation was required, as demonstrated by our similar CAE rate $(3.3 \%)$ to prior published studies [27].

Lastly, we did not have records on patient's satisfaction and propofol dosages separately between scope intubation and withdrawal periods, which could have assisted to clarify our theory on the advantages of endoscopist-directed sedation over the anesthesiologist-administered method.

Our results supports the recommendation of many gastrointestinal societies [32], that sedation administered by nurses under endoscopist supervision could become a standard of general endoscopy practice, requiring that both endoscopists and nurses be properly trained in sedation and resuscitation techniques.

\section{CONCLUSIONS}

Our study highlighted the efficacy, and safety of NAPS as it requires a significantly lower dosage of propofol without the difference in the success rate or procedural time for low-risk routine colonoscopy. Given the increasing health care costs and lack of availability or superiority of OAPS, sedation for routine colonoscopy administered by the anesthesiologist in low-risk patients now appears to be increasingly unsustainable. Further randomized controlled studies with larger cohorts are warranted to strengthen our findings and cement the role of NAPS in routine colonoscopy in the future.

\section{Conflicts of interest: None to declare.}

Authors' contribution: K.T. and R.R. conceived and designed the study and drafted the manuscript. K.T., P.M., R.P., S.G., P.K., R.R. collected and analyzed the data. K.T., P.K., R.R. critically revised the manuscript. All authors agreed on the content of the manuscript and approved the final version on the manuscript.

\section{REFERENCES}

1. Standards of Practice Committee of the American Society for Gastrointestinal Endoscopy; Lichtenstein DR, Jagannath S, et al. Sedation and anesthesia in GI endoscopy. Gastrointest Endosc 2008;68:815-826. doi:10.1016/j.gie.2008.09.029

2. Lin OS. Sedation for routine gastrointestinal endoscopic procedures: a review on efficacy, safety, efficiency, cost and satisfaction. Intest Res 2017;15:456-466. doi:10.5217/ir.2017.15.4.456

3. Vargo JJ, Cohen LB, Rex DK, et al. Position statement: Nonanesthesiologist administration of propofol for GI endoscopy. Gastroenterology 2009;137:2161-2167. doi:10.1053/j.gastro.2009.09.050

4. Kayaalti S, Kayaalti O. Safety of applying midazolam-ketamine-propofol sedation combination under the supervision of endoscopy nurse with patient-controlled analgesia pump in colonoscopy. World J Clin Cases 2018;6:1146-1154. doi:10.12998/wjcc.v6.i16.1146

5. Dumonceau JM, Riphaus A, Aparicio JR, et al. European Society of Gastrointestinal Endoscopy, European Society of Gastroenterology and Endoscopy Nurses and Associates, and the European Society of Anaesthesiology Guideline: Non-anesthesiologist administration of propofol for GI endoscopy. Endoscopy 2010;42:960-974. doi:10.1055/s-0030-1255728

6. Cohen LB, Hightower CD, Wood DA, Miller KM, Aisenberg J. Moderate level sedation during endoscopy: a prospective study using low-dose propofol, meperidine/fentanyl, and midazolam. Gastrointest Endosc 2004;59:795-803. doi:10.1016/s0016-5107(04)00349-9

7. ASGE Standards of Practice Committee, Early DS, Lightdale JR, et al. Guidelines for sedation and anesthesia in GI endoscopy. Gastrointest Endosc 2018;87:327-337. doi:10.1016/j.gie.2017.07.018

8. McQuaid KR, Laine L. A systematic review and meta-analysis of randomized, controlled trials of moderate sedation for routine endoscopic procedures. Gastrointest Endosc 2008;67:910-923. doi:10.1016/j.gie.2007.12.046

9. Perel A. Non-anaesthesiologists should not be allowed to administer propofol for procedural sedation: a Consensus Statement of 21 European National Societies of Anaesthesia. Eur J Anaesthesiol 2011;28:580-584. doi:10.1097/EJA.0b013e328348a977

10. Ooi M, Thomson A. Morbidity and mortality of endoscopistdirected nurse-administered propofol sedation (EDNAPS) in a tertiary referral center. Endosc Int Open. 2015;3:E393-E397. doi:10.1055/s-0034-1392511

11. Jensen JT, Hornslet P, Konge L, Moller AM, Vilmann P. High efficacy with deep nurse-administered propofol sedation for advanced gastroenterologic endoscopic procedures. Endosc Int Open 2016;4:E107-E111. doi:10.1055/s-0041-107899

12. Rivera B. The Current Status of Nurse-Administered Propofol Sedation in Endoscopy: An Evidence-Based Practice Nurse Fellowship Project. Gastroenterol Nurs 2015;38:297-304. doi:10.1097/ SGA.0000000000000099

13. Rex DK. Effect of the Centers for Medicare \& Medicaid Services policy about deep sedation on use of propofol. Ann Intern Med 2011;154:622626. doi:10.7326/0003-4819-154-9-201105030-00007

14. Aisenberg J, Cohen LB, Piorkowski JD Jr. Propofol use under the direction of trained gastroenterologists: an analysis of the medicolegal implications. Am J Gastroenterol 2007;102:707-713.

15. Riphaus A, Macias-Gomez C, Deviere J, Dumonceau JM. Propofol, the preferred sedation for screening colonoscopy, is underused. Results of an international survey. Dig Liver Dis 2012;44:389-392. doi:10.1016/j. dld.2011.10.019 
16. Inadomi JM, Gunnarsson CL, Rizzo JA, Fang H. Projected increased growth rate of anesthesia professional-delivered sedation for colonoscopy and EGD in the United States: 2009 to 2015. Gastrointest Endosc 2010;72:580-586. doi:10.1016/j.gie.2010.04.040

17. Ruiz-Curiel RE, Bonilla HY, Baptista A, Bronstein M. Sedation with propofol in digestive endoscopy administered by gastroenterologists. Experience in a Venezuelan hospital. Rev Esp Enferm Dig 2018;110:246249. doi:10.17235/reed.2018.5185/2017

18. Vargo JJ, Niklewski PJ, Williams JL, Martin JF, Faigel DO. Patient safety during sedation by anesthesia professionals during routine upper endoscopy and colonoscopy: an analysis of 1.38 million procedures. Gastrointest Endosc 2017;85:101-108. doi:10.1016/j.gie.2016.02.007

19. Cooper GS, Kou TD, Rex DK. Complications following colonoscopy with anesthesia assistance: a population-based analysis. JAMA Intern Med 2013;173:551-556. doi:10.1001/jamainternmed.2013.2908

20. Wernli KJ, Brenner AT, Rutter CM, Inadomi JM. Risks Associated With Anesthesia Services During Colonoscopy. Gastroenterology 2016;150:888-894. doi:10.1053/j.gastro.2015.12.018

21. Rex DK, Deenadayalu VP, Eid E, et al. Endoscopist-directed administration of propofol: a worldwide safety experience. Gastroenterology 2009;137:1229-1237. doi:10.1053/j.gastro.2009.06.042

22. Hassan C, Rex DK, Cooper GS, Benamouzig R. Endoscopist-directed propofol administration versus anesthesiologist assistance for colorectal cancer screening: a cost-effectiveness analysis. Endoscopy 2012;44:456 464. doi:10.1055/s-0032-1308936

23. Committee on Quality Management and Departmental Administration. Continuum of Depth of Sedation: Definition of General Anesthesia and Levels of Sedation/Analgesia. Anesthesiologists October 23, 2019.

24. Jensen JT, Vilmann P, Horsted T, et al. Nurse-administered propofol sedation for endoscopy: a risk analysis during an implementation phase. Endoscopy 2011;43:716-722. doi:10.1055/s-0030-1256515
25. Mason KP, Green SM, Piacevoli Q, International Sedation Task F Adverse event reporting tool to standardize the reporting and tracking of adverse events during procedural sedation: a consensus document from the World SIVA International Sedation Task Force. Br J Anaesth 2012;108:13-20. doi:10.1093/bja/aer407

26. Poincloux L, Laquiere A, Bazin JE, et al. A randomized controlled trial of endoscopist vs. anaesthetist-administered sedation for colonoscopy. Dig Liver Dis 2011;43:553-558. doi:10.1016/j.dld.2011.02.007

27. Sieg A; bng-Study-Group, Beck S, et al. Safety analysis of endoscopistdirected propofol sedation: a prospective, national multicenter study of 24441 patients in German outpatient practices. J Gastroenterol Hepatol 2014;29:517-523. doi:10.1111/jgh.12458

28. Daza JF, Tan CM, Fielding RJ, Brown A, Farrokhyar F, Yang I. Propofol administration by endoscopists versus anesthesiologists in gastrointestinal endoscopy: a systematic review and meta-analysis of patient safety outcomes. Can J Surg 2018;61:226-236. doi:10.1503/ cjs.008117

29. Ferreira AO, Torres J, Barjas E, et al. Non-anesthesiologist administration of propofol sedation for colonoscopy is safe in low risk patients: results of a noninferiority randomized controlled trial. Endoscopy 2016;48:747753. doi:10.1055/s-0042-105560

30. Goudra BG, Singh PM, Gouda G, et al. Safety of Non-anesthesia ProviderAdministered Propofol (NAAP) Sedation in Advanced Gastrointestinal Endoscopic Procedures: Comparative Meta-Analysis of Pooled Results. Dig Dis Sci 2015;60:2612-2627. doi:10.1007/s10620-015-3608-X

31. Aisenberg J, Brill JV, Ladabaum U, Cohen LB. Sedation for gastrointestinal endoscopy: new practices, new economics. Am J Gastroenterol 2005;100:996-1000.

32. Vargo JJ. Propofol may be safely administered by trained nonanesthesiologists. Pro: Propofol demystified: it is time to change the sedation paradigm. Am J Gastroenterol. 2004;99:1207-1208. 